\title{
The clinical outcomes of fresh versus frozen embryos transfer in women $\geq 40$ years with poor ovarian response
}

\author{
Conghui Liu, PhD, Yu Li, MD, Hong Jiang, PhD, Yingchun Liu, MD, Xiaomin Song, MD \\ Reproductive Medicine Center, The 901 th Hospital of the Joint Logistics Support Force of People's Liberation Army, Hefei, China
}

\section{Objective}

To compare the clinical outcomes of fresh embryo transfers (ETs) and frozen-thawed embryo transfers (FETs) after a freeze-all cycle in women $\geq 40$ years old with poor ovarian response (POR).

Methods

We performed a single-center, retrospective, case-control study of patients who underwent in vitro fertilization between January 2014 and June 2019. We included a total of 192 patients aged 40 years or older from whom 3 or fewer oocytes had been retrieved and who were receiving cleavage-stage ET in this study. Of these patients, 101 and 91 patients underwent fresh ET and FET, respectively. The primary outcome was the live birth rate (LBR) after the first ET. Logistic regression analysis was used to compare the IVF outcomes and neonatal characteristics between the fresh ET and FET groups, adjusting for maternal age, body mass index, luteinizing hormone, and the number of good quality embryos transferred.

\section{Results}

The mean maternal ages and number of oocytes retrieved (43.2 years and 2.3 in both groups, $P=0.902$ and $P=0.927$, respectively) were similar in the fresh ET and FET groups. No significant difference was observed between the LBRs of the fresh ET and FET groups (adjusted odds ratio, 1.28; 95\% confidence interval, 0.29-5.70). The clinical pregnancy and miscarriage rates, and neonatal characteristics (birth weights and premature infant rates) were similar between the 2 groups.

Conclusions

FET after the freeze-all strategy had no beneficial impact on the clinical outcomes of women $\geq 40$ years with POR.

Keywords: Maternal age; Infertility; In vitro fertilization; Cryopreservation; Embryo transfer

\section{Introduction}

With the development of in vitro fertilization (IVF) techniques over the past decades, clinicians are constantly seeking ways to optimize IVF outcomes and minimize the treatment risks [1]. The "freeze-all" strategy, which involves the freezing of all of the embryos in a fresh cycle and subsequent frozenthawed embryo transfer (FET), has been reported to increase IVF efficacy and has become an essential aspect of assisted reproductive technology (ART) [2].

In the last century, there have been extensive studies that have compared the IVF outcomes of fresh embryo transfer (ET) and FET [3]. A previous randomized controlled trial (RCT) from China indicated that patients with polycystic ovary syndrome (PCOS) who underwent FET had a higher live birth rate (LBR), and lower risks of ovarian hyperstimulation syndrome (OHSS) and pregnancy loss compared with patients

Received: 2020.05.08. Revised: 2020.11.24. Accepted: 2020.12.10. Corresponding author: Hong Jiang, $\mathrm{PhD}$

Reproductive Medicine Center, The 901th Hospital of the Joint Logistics Support Force of People's Liberation Army, 424 West Changjiang Rd, Hefei, Anhui 230031, China

E-mail: jiangh105@sina.com

https://orcid.org/0000-0002-4690-7906

Articles published in Obstet Gynecol Sci are open-access, distributed under the terms of the Creative Commons Attribution Non-Commercial License (http://creativecommons. org/licenses/by-nc/3.0/) which permits unrestricted non-commercial use, distribution, and reproduction in any medium, provided the original work is properly cited.

Copyright $\odot 2021$ Korean Society of Obstetrics and Gynecology 


\section{Obstetrics \& Gynecology Science}

Conghui Liu, et al. Aged POR women will not benefit from FET

who underwent fresh ET. However, whether the strategy is suitable for other patients who are undergoing IVF, such as women with advanced age or poor ovarian response (POR) is yet to be elucidated [4]. In contrast, according to a recent meta-analysis of 11 RCTs that included 5,379 patients, the significant increase in the FET LBR was only noted in hyperresponders with an increased risk of pre-eclampsia; however, the researchers did not analyze the differences among the patients with POR [5].

Recently, a few observational studies compared the fresh ET and FET outcomes in poor ovarian responders and the results were equivocal. A retrospective cohort study that included 82,935 cycles showed that both the clinical pregnancy rate (CPR) and LBR were higher after fresh ET compared with FET in intermediate (6-14 oocytes retrieved; $49.6 \%$ vs. $44.2 \%$ and $41.2 \%$ vs. $35.3 \%$, respectively) and low responders (1-5 oocytes retrieved; $33.2 \%$ vs. $15.9 \%$ and $25.9 \%$ vs. $11.5 \%$ ). However, the results derived from both the cleavage-stage embryo and blastocyst transfer data exhibited high heterogeneity [6]. Berkkanoglu et al. [7] demonstrated that the freeze-all strategy should be made available to poor responders as it resulted in improved pregnancy outcomes; however, the mean age in their study was only 35 years old with 4 or fewer oocytes collected, which did not meet the POR criteria proposed by Bologna [8]. In contrast, another retrospective cohort study from Brazil demonstrated that the freeze-all strategy had no impact on IVF outcomes in POR patients; however, the primary outcome in their study was the ongoing pregnancy rate but not the LBR [9].

To the best of our knowledge, there are no studies that have previously evaluated the clinical outcomes of fresh ET and FET after use of the freeze-all strategy in advanced maternal age (AMA) patients with POR. This study aimed to investigate whether AMA patients with POR can benefit from the freeze-all strategy.

\section{Materials and methods}

\section{Study population}

This was a retrospective case-control study that analyzed the data of AMA patients with POR who underwent fresh ET and FET after the "freeze-all" strategy from January 2014 to June 2019 at the Reproductive Centre of the 901th Hospital of the Joint Logistics Support Force of The People's Liberation Army.
All the patients provided written informed consent. The eligibility criteria were as follows: 1 ) aged 40 years or older; 2 ) 3 or less oocytes retrieved after controlled ovarian stimulation (COS) including the gonadotropin-releasing hormone $(\mathrm{GnRH})$ antagonist, GnRH agonist short, mild stimulation, and medroxyprogesterone acetate (MPA) pituitary downregulation protocols [10]; 3) at least one available embryo for transfer after COS; 4) had undergone cleavage-stage ET. The exclusion criteria were: 1) oocyte donation cycles; 2) embryo biopsy; 3) uterine malformations; 4) intrauterine adhesions. None of the patients were excluded due to male factors. The subjects assigned to the FET group were mostly those who received the MPA pituitary down-regulation protocol (54.9\%) and who had an endometrial thickness that was less than $8 \mathrm{~mm}$ (13.2\%; Table 1).

A total of 192 patients who met the criteria were included in this study. Of these patients, 101 and 91 received fresh ETs and FETs after undergoing the freeze-all strategy, respectively. We collected the patients' demographic information from medical records, including their age; infertility duration and type; body mass index (BMI); basic follicle-stimulating hormone $(\mathrm{FSH})$, luteinizing hormone $(\mathrm{LH})$, estradiol $\left(\mathrm{E}_{2}\right)$, and prolactin levels; and antral follicle count (AFC).

\section{Ovarian stimulation}

All of the patients received COS. The starting gonadotropin dose was 150-300 IU FSH based on the patient's age, hormone profile, AFC, and BMI. The dose was adjusted according to the ovarian response through serial ultrasonography and serum FSH, $\mathrm{LH}, \mathrm{E}_{2}$, and progesterone level monitoring. The doctors would add 75 IU human menopausal gonadotropin (Lizhu Pharmaceutical Trading Co., Ltd., Zhuhai, China) at their discretion. When one or 2 leading follicles reached

Table 1. Reason for frozen embryo transfer $(n=91)$

\begin{tabular}{lc}
\hline Reasons & Value \\
\hline MPA pituitary down-regulation protocol & $50(54.9)$ \\
Endometrial thickness less than $8 \mathrm{~mm}$ & $12(13.2)$ \\
AMA per se & $11(12.1)$ \\
Level of progesterone over $1.5 \mathrm{ng} / \mathrm{mL}$ & $9(9.9)$ \\
Personal choice & $5(5.5)$ \\
Others & $4(4.4)$ \\
\hline
\end{tabular}

Values are presented as number (\%).

MPA, medroxyprogesterone acetate; AMA, advanced maternal age. 


\section{Obstetrics \& Gynecology Science}

Vol. 64, No. 3, 2021

$18 \mathrm{~mm}$ or more in diameter, $250 \mu \mathrm{g}$ recombinant human chorionic gonadotropin (The Merck Group, Darmstadt, Germany) was administered to induce oocyte maturation. All the oocytes that were $\geq 12 \mathrm{~mm}$ in diameter were transvaginally retrieved 36 hours later.

\section{Embryo culture and evaluation}

All of the oocytes that were retrieved were fertilized with conventional IVF or intracytoplasmic sperm injection (ICSI) 2-4 hours after oocyte retrieval was performed, according to the sperm quality. The embryos were graded according to the system proposed by Cummins et al. [11] The available embryos were defined as normally fertilized embryos with 6 or more blastomeres and $<50 \%$ fragmentation on day 3 . Good quality embryos were defined as those with a grade equal or superior to $7 / \|$ on day 3 .

\section{Embryo transfer and luteal support}

For the patients who received fresh ETs, $\leq 3$ available embryos were transferred into the uterine cavity under ultrasonographic guidance on day 3 after the oocyte retrieval. A daily intramuscular injection of $40 \mathrm{mg}$ progesterone (Zhejiang Xianju Pharmaceutical Co Ltd., Taizhou, China) was used for luteal support from the day of oocyte retrieval until 10 weeks of gestation after clinical pregnancy was confirmed (defined as the presence of a gestational sac on ultrasound 30-35 days after ET).

For the patients who underwent FET, hormone replacement treatment was carried out for endometrial preparation. Briefly, 4-8 mg oral $E_{2}$ valerate tablets (Progynova; Bayer Schering Pharma, Beijing, China) were administered from day 3 of the second menstrual cycle after oocyte retrieval or later. An intramuscular progesterone injection at a dose of $40 \mathrm{mg}$ per day was added for 3 days when the endometrial thickness reached $8 \mathrm{~mm}$ or more. For patients that had an endometrial thickness that was less than $8 \mathrm{~mm}$ after prolonged oral $E_{2}$ valerate tablet usage (18 days) and that did not have any disorders identified by hysteroscopy, endometrial transformation with $40 \mathrm{mg}$ progesterone were also performed. The frozen embryos were thawed with a commercial warming kit (Kitazato Biopharma Co., Yanagishima, Japan), and $\leq 3$ surviving embryos (defined by at least $50 \%$ retention of intact cells) were transferred into the uterine cavity under ultrasonographic guidance. Luteal support with the original $E_{2}$ valerate dose and intramuscular progesterone injections were continued until 10 weeks of gestation once clinical pregnancy was confirmed.

\section{Outcomes}

The primary outcome in this study was the LBR. Live birth was defined as the birth of at least one newborn that exhibited any signs of life. The secondary outcomes were the biochemical pregnancy, clinical pregnancy, and miscarriage rates. Biochemical pregnancy was defined as an human chorionic gonadotropin level more than 20 IU/L 14 days after ET. Miscarriage was defined as the loss of a clinical pregnancy at $<24$ weeks' gestation.

The mean birth weights, premature infant rates, number of multiple pregnancies, and numbers of neonates with low birth weights and fetal macrosomia were recorded. A premature infant was defined as preterm birth at $<37$ weeks' gestation. Multiple pregnancy was defined as at least 2 detectable gestational sacs or heartbeats on ultrasound. Low birth weight and macrosomia were defined as birth weights $<2,500 \mathrm{~g}$ and $\geq 4,000 \mathrm{~g}$, respectively.

\section{Statistical analysis}

Continuous variables are expressed as the mean with the standard deviation or the median with the interquartile range, categorical variables are expressed as numbers (percentages). Comparisons between the patients who underwent fresh ET and FET were performed using the $t$-test for continuous variables when the distribution and variance met the conditions. Otherwise, the Kruskal-Wallis test was used.

To identify the differences in the reproductive and neonatal characteristics between the fresh ET and FET groups, multivariate logistic regression analysis was used to derive the odds ratios (ORs) and 95\% confidence intervals ( $\mathrm{Cls}$ ). The potential confounders were variables that resulted in more than $10 \%$ variation in the coefficient of the principal study factor when added into the model. Results were presented after adjusting for maternal age, BMI (model 1), LH, and the number of good quality embryos transferred (model 2).

A 2-tailed $P$-value that was less than 0.05 was considered statistically significant. All analyses were performed using STATA 16.0 (StataCorp, College Station, TX, USA). 


\section{Obstetrics \& Gynecology Science}

Conghui Liu, et al. Aged POR women will not benefit from FET

\section{Results}

\section{Patient characteristics}

The patient characteristics are shown in Table 2. There were no significant differences in the maternal ages; infertility durations and types; basal FSH, $\mathrm{LH}, \mathrm{E}_{2}$, and prolactin levels; and AFCs between the fresh ET and FET groups. However, the BMI was higher for patients in the fresh ET group compared with the FET group $\left(25.0 \mathrm{~kg} / \mathrm{m}^{2} \mathrm{vs} .23 .9 \mathrm{~kg} / \mathrm{m}^{2}, P=0.044\right)$.

\section{Controlled ovarian stimulation outcomes}

The outcomes are shown in Table 3. The number of days of ovarian stimulation and gonadotropin dose were similar be- tween the fresh ET and FET groups. No significant differences were identified between the numbers of oocytes retrieved, available embryos, and good quality embryos between the 2 groups. Although the numbers of embryos transferred were similar between the 2 groups, the FET group had a greater number of good quality embryos transferred and had thinner endometria compared with the fresh ET group (1.6 vs. 0.9 and $7.9 \mathrm{~mm}$ vs. $9.6 \mathrm{~mm}$, respectively; $P<0.001$ for both).

\section{In vitro fertilization and neonatal outcomes}

Table 4 shows that the LBRs were similar between the fresh ET and FET groups (5.9\% vs. 6.6\%). When the FET and fresh ET groups were compared, the unadjusted OR was $1.12(95 \%$

Table 2. Characteristics of patients

\begin{tabular}{|c|c|c|c|}
\hline Characteristics & Fresh ET group $(n=101)$ & Frozen ET group $(n=91)$ & $P$-value \\
\hline Age (yr) & $43.2 \pm 2.6$ & $43.2 \pm 2.2$ & 0.902 \\
\hline Infertility duration (yr) & $4.9 \pm 4.7$ & $5.7 \pm 4.9$ & 0.276 \\
\hline \multicolumn{4}{|l|}{ Infertility type } \\
\hline Primary infertility & $13(12.9)$ & $12(14.5)$ & 0.755 \\
\hline Secondary infertility & $88(87.1)$ & $71(85.5)$ & \\
\hline $\operatorname{BMI}\left(\mathrm{kg} / \mathrm{m}^{2}\right)$ & $25.0 \pm 4.1$ & $23.9 \pm 3.1$ & 0.044 \\
\hline Basal FSH (IU/L) & $13.1 \pm 7.3$ & $13.7 \pm 9.2$ & 0.668 \\
\hline Basal LH (IU/L) & $5.2 \pm 3.2$ & $6.5 \pm 6.3$ & 0.087 \\
\hline Basal oestrogen (pg/mL, median [IQR]) & $47.5(53)$ & $55.5(63.5)$ & 0.513 \\
\hline Basal prolactin (ng/mL, median [IQR]) & $11.2(7.1)$ & $12.0(9.0)$ & 0.598 \\
\hline AFC & $4.9 \pm 3.1$ & $4.7 \pm 3.7$ & 0.673 \\
\hline
\end{tabular}

Values are presented as mean \pm standard deviation or number (\%) unless otherwise indicated.

ET, embryo transfer; BMI, body mass index; FSH, follicle stimulating hormone; LH, luteinizing hormone; IQR, interquartile range; AFC, antral follicle count.

Table 3. The outcomes of controlled ovarian stimulation

\begin{tabular}{lccc}
\hline Characteristics & Fresh ET group & Frozen ET group & $\boldsymbol{P}$-value \\
\hline Days of ovarian stimulation (day) & $7.7 \pm 3.2$ & $7.6 \pm 3.3$ & 0.804 \\
Total gonadotropin dose (IU) & $1,933.8 \pm 877.2$ & $1,911.9 \pm 1,054.2$ & 0.877 \\
Endometrial thickness (mm) & $9.6 \pm 2.6$ & $7.9 \pm 2.7$ & $2.3 \pm 0.7$ \\
Oocytes retrieved & $2.3 \pm 0.7$ & $1.9 \pm 0.6$ & 0.927 \\
Available embryos & $1.8 \pm 0.7$ & $1.6 \pm 0.7$ & 0.616 \\
Good quality embryos & $1.5 \pm 0.8$ & $1.9 \pm 0.5$ & 0.396 \\
Embryos transferred & $1.8 \pm 0.7$ & $1.6 \pm 0.6$ & 0.687 \\
Good quality embryo transferred & $0.9 \pm 0.8$ & $<0.001$ & \\
\hline
\end{tabular}

Values are presented as mean \pm standard deviation.

ET, embryo transfer. 


\section{Obstetrics \& Gynecology Science}

Vol. 64, No. 3, 2021

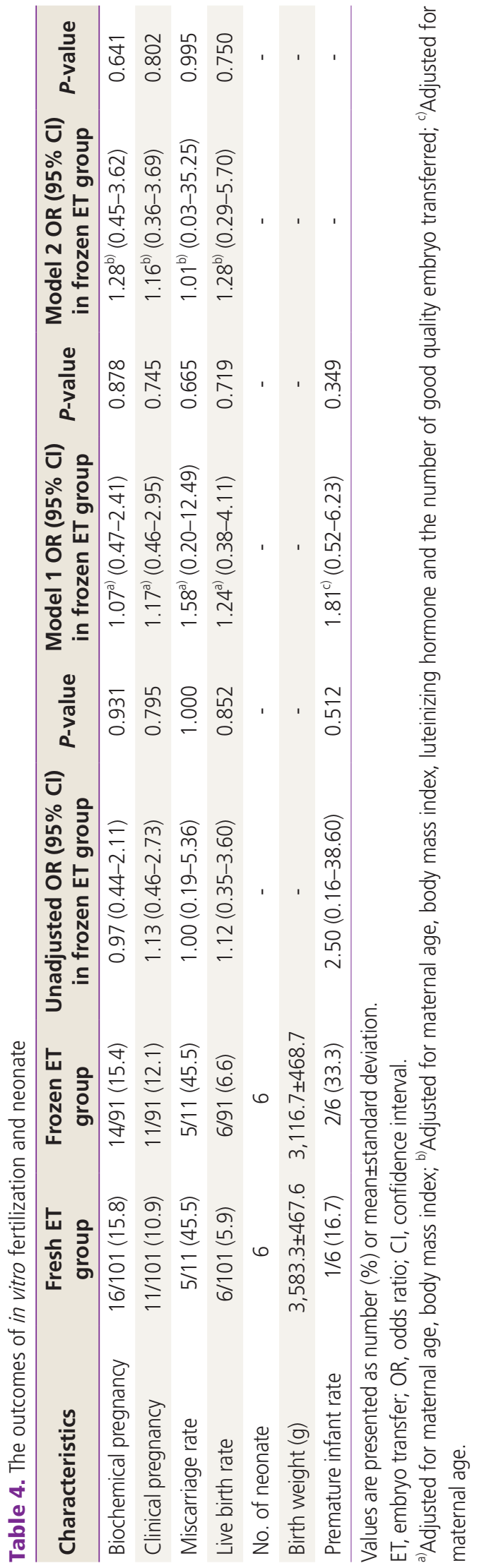

$\mathrm{Cl}, 0.35-3.60)$. After adjusting for the age and $\mathrm{BMI}$, the OR rose $1.24(95 \% \mathrm{Cl}, 0.38-4.11)$, but this value was still not statistically significant. After further adjusting for $\mathrm{LH}$ and the number of good quality embryos transferred, there was little difference in the $\mathrm{OR}(\mathrm{OR}, 1.28 ; 95 \% \mathrm{Cl}, 0.29-5.70)$. There were no significant differences between the groups regarding the biochemical pregnancy, clinical pregnancy, and miscarriage rates before and after the adjustment.

Six neonates were born after fresh ET and FET each. The mean birth weights were 3,583.3 $\mathrm{g}$ and 3,116.7 $\mathrm{g}$ in the fresh ET and FET groups, respectively $(P=0.115)$. The premature infant rate was higher in the FET group compared with the fresh ET group, but this difference was not statistically significant (33.3\% vs. $16.7 \%$ ). The adjusted OR was 1.81 in the FET group when compared with the fresh ET group (95\% $\mathrm{Cl}, 0.52-6.23 ; P=0.349)$. Neither of the groups had low birth weight or macrosomic neonates (Table 4).

\section{Discussion}

With the rapid increase in the success rate with vitrifiedthawed embryos, the number of autologous FETs increased over 2.5-fold from 2004 to 2013; however, the number of fresh ETs was approximately the same [12]. The increased use of FET corresponded with a significant increase in the LBR (from 16.9\% in 2006 to $31.5 \%$ in 2012) and a decrease in the OHSS risk, especially for women with PCOS $[4,13]$. The potential mechanism for the decreased LBR after fresh ET, when compared with FET, could be the supraphysiologic hormonal levels during COS resulting in endometrial implantation window advancement and alterations in the endometrial gene expression compared with the natural cycle $[12,14,15]$. In contrast with the previous study, the LBR in this study was comparable between the fresh ET and FET groups (adjusted $\mathrm{OR}, 1.28 ; 95 \% \mathrm{Cl}, 0.29-5.70 ; P=0.750)$. The possible explanation for this is that, here, the patients were 40 years or older and had POR; thus the $E_{2}$ levels in the fresh ET cycle were not high. This resulted in fewer changes to the internal environment and less endometrial receptivity in the ovarian stimulation process.

Although many studies have illustrated that IVF outcomes (LBR, implantation rate, and ongoing pregnancy) can be improved through the use of the freeze-all strategy, most of these studies were limited by the number of patients who 


\section{Obstetrics \& Gynecology Science}

Conghui Liu, et al. Aged POR women will not benefit from FET

were hyper- or normal ovarian responders $[5,16,17]$. An RCT on normal responders (8-15 AFC), that included patients with an average age of 33 years old, showed that the clinical and ongoing pregnancy rates in the frozen ET group were significantly greater than those in the fresh ET group [18]. Recently, a multicenter RCT that included 1,508 PCOS patients was conducted in China. The authors demonstrated that patients who underwent frozen ET had higher LBRs than those who underwent fresh ET ( $49.3 \%$ vs. $42.0 \%$; rate ratio, $1.17 ; 95 \% \mathrm{Cl}, 1.05-1.31)$ with no differences in the neonatal complications; however, 5 neonatal deaths did happen in the frozen ET group [4]. They performed another study of patients aged approximately 29 years old $(n=1,650)$ who had regular menstrual cycles and who underwent elective single blastocyst transfers. Their study also showed a higher LBR in the frozen ET group compared with the fresh ET group; however, the frozen ET group had a higher pre-eclampsia risk (3.1\% vs. 1.0\%) [17]. Nevertheless, in patients with POR, previous studies have had inconsistent results and have been unclear about the benefits of the freeze-all strategy. A previous cohort study that included 938 IVF cycles suggested that only patients that had 10-15 oocytes retrieved had higher implantation and ongoing pregnancy rates after FET ( $P=0.028$ and $P=0.021$, respectively) compared with fresh $E T$, while in patients that had 4-9 oocytes retrieved, the implantation and ongoing pregnancy rates $(P=0.259$ and $P=0.577$, respectively) did not improve from FET after use of the freeze-all strategy, which suggests that the potential advantages of the freeze-all strategy decrease with worsening ovarian response [19]. Furthermore, a meta-analysis of 5 RCTs concluded that the freeze-all strategy was not advantageous when the mean number of oocytes retrieved was less than 15 [20]. To date, there have been no studies that have reported on the efficiency of frozen ET for AMA patients with POR, and whether the freeze-all strategy is suitable for all patients, irrespective of their age, ovarian response, and hormone levels during COS remains unknown [21].

POR, first described by Garcia et al. [22], represents a challenging group of patients who undergo IVF and the prognosis differs between older poor responders and young women $[5,23,24]$. Our previous study of patients older than 40 years demonstrated women without POR had a relatively higher cumulative LBR compared with women with POR, indicating that ovarian reserves play a significant role in female fertility [25]. Thus far, the clinical outcomes of POR and AMA patients when choosing between fresh ET and FET remain inconsistent. A retrospective cohort study of 2,263 patients who received ICSIs demonstrated that patients who had 4 or less oocytes retrieved could also benefit from the freezeall strategy, with better pregnancy outcomes including the pregnancy, clinical pregnancy, implantation, and LBRs compared with those who underwent fresh ET. However, the mean age in this study was 35 years old or younger, which might not have been representative of the older population as a whole [7]. In contrast, another case-control study of 259 POR patients with an average age of 36.9 years (range, 21-43 years) showed a similar LBR between the fresh ET and FET groups ( $21 \%$ vs. $17 \%, P=0.42$ ); however, they did not consider some of the related confounders such as the BMI [26]. In this study, all the subjects were 40 years old or older, with a mean age of 43.2 years old and mean number of oocytes retrieved of 2.3 in both the fresh ET and FET groups, which was a more rational representation of older women with POR. The results showed that there were no significant differences in terms of the CPR, LBR, and neonatal characteristics (body weight and premature infant rate) between the fresh ET and FET groups, indicating that the freeze-all strategy holds no benefit over fresh ET in AMA patients with POR who are undergoing cleavage-stage ET. The results were in accordance with those from previous studies that found that the benefits of the freeze-all strategy decreased as the number of oocytes retrieved decreased.

As an independent factor for ART outcomes, age predicts over $80 \%$ of IVF success [27]. Even in poor ovarian responders, being young can act as a protective factor against the deleterious effects of POR [24]. The most probable reason for the steep decline in fertility with aging is a diminished ovarian reserve [27]. Moreover, among women with advanced age, a significant increase in the number of aneuploidy oocytes and a decrease in the potential for embryonic development are also noticeable. The number of antral follicles and the follicular microenvironment (the granulosa cells and vasculature) are also negatively affected by age $[28,29]$. The best assisted reproductive strategy for AMA patients is to shorten the time to get pregnant. However, the first FET with the freezeall strategy is usually performed 2 or 3 months after oocyte retrieval, and the median time to conception is 1.4 months later compared with the fresh ET cycle [30]. In addition, the cryopreservation may have some disadvantages such as the cost, time, inconvenience, and possible alterations in the 


\title{
Obstetrics \& Gynecology Science
}

\author{
Vol. 64, No. 3, 2021
}

embryo's competence for implantation and/or development derived from membrane damage and general protein denaturation in the process of embryo freezing and thawing [20]. Furthermore, FET is associated with the emotional costs of deferring ET and physical costs of additional treatments such as hormone administration, multiple injections, and medical consultations [31]. In this study, AMA patients with POR did not benefit from the freeze-all strategy, indicating there is no need to replace fresh ET with FET after use of the freeze-all strategy.

There are several limitations to this study. First, the retrospective design had inherent problems relating to selection bias; thus, the characteristics and prognoses of the patients assigned to the study may have differed between the 2 groups. However, as shown in Table 1, the patient characteristics were similar between the fresh ET and FET groups. Second, the study had a relatively small sample size; therefore, the results should be interpreted carefully. Reassuringly, the similarities in the findings regarding the biochemical pregnancy, clinical pregnancy, and miscarriage rates between the fresh ET and FET groups support our findings. Unquestionably, an RCT with a large sample size should be carried out to further examine whether the freeze-all strategy can be beneficial to AMA patients with POR. Finally, there was a significant difference between the 2 groups when analyzing the endometrial thickness and number of good quality embryos transferred. However, we only found $2.3 \%$ variation in the coefficient of the principal study factor when endometrial thickness was added into the logistic regression model; therefore we did not adjust for it. We had already excluded patients with intrauterine adhesions; thus, the influence of endometrial thickness on the clinical outcomes in this study may not be as significant as it appears. Regarding the number of good quality embryos transferred, the variation in the coefficient was $55.5 \%$ when added it into the model with no interaction (effect modification) between the different numbers. Thus, we did not perform a stratification analysis of the number of good quality embryos transferred. Moreover, stratification will further decrease the sample size in each layer, resulting in lower statistical power.

In conclusion, compared with fresh ET, FET followed by a freeze-all cycle is not beneficial for women $\geq 40$ years old with POR. RCTs with a large sample size should be carried out to confirm this conclusion.

\section{Acknowledgments}

The authors would like to thank the Reproductive Centre of the 901th Hospital of the Joint Logistic Support Force of People's Liberation Army, as well as all its members of staff, and the patients who allowed for their data to be reported.

\section{Conflict of interest}

No potential conflict of interest relevant to this article was reported.

\section{Ethical approval}

All the patients provided written informed consent.

\section{Patient consent}

All the patients provided written informed consent.

\section{Funding information}

None.

\section{References}

1. La Marca A, Sunkara SK. Individualization of controlled ovarian stimulation in IVF using ovarian reserve markers: from theory to practice. Hum Reprod Update 2014;20:124-40.

2. Rienzi L, Gracia C, Maggiulli R, LaBarbera AR, Kaser DJ, Ubaldi FM, et al. Oocyte, embryo and blastocyst cryopreservation in ART: systematic review and meta-analysis comparing slow-freezing versus vitrification to produce evidence for the development of global guidance. Hum Reprod Update 2017;23:139-55.

3. Ferraretti AP, Gianaroli L, Magli C, Fortini D, Selman HA, Feliciani E. Elective cryopreservation of all pronucleate embryos in women at risk of ovarian hyperstimulation syndrome: efficiency and safety. Hum Reprod 


\section{Obstetrics \& Gynecology Science}

Conghui Liu, et al. Aged POR women will not benefit from FET

1999;14:1457-60.

4. Chen ZJ, Shi $Y$, Sun $Y$, Zhang $B$, Liang $X$, Cao $Y$, et al. Fresh versus frozen embryos for infertility in the polycystic ovary syndrome. N Engl J Med 2016;375:523-33.

5. Roque M, Haahr T, Geber S, Esteves SC, Humaidan P. Fresh versus elective frozen embryo transfer in IVF/ICSI cycles: a systematic review and meta-analysis of reproductive outcomes. Hum Reprod Update 2019;25:2-14.

6. Acharya KS, Acharya CR, Bishop K, Harris B, Raburn D, Muasher SJ. Freezing of all embryos in in vitro fertilization is beneficial in high responders, but not intermediate and low responders: an analysis of 82,935 cycles from the Society for Assisted Reproductive Technology registry. Fertil Steril 2018;110:880-7.

7. Berkkanoglu M, Coetzee K, Bulut H, Ozgur K. Optimal embryo transfer strategy in poor response may include freeze-all. J Assist Reprod Genet 2017;34:79-87.

8. Ferraretti AP, La Marca A, Fauser BC, Tarlatzis B, Nargund $G$, Gianaroli $L$, et al. ESHRE consensus on the definition of 'poor response' to ovarian stimulation for in vitro fertilization: the Bologna criteria. Hum Reprod 2011;26:1616-24.

9. Roque M, Valle M, Sampaio M, Geber S. Does freeze-all policy affect IVF outcome in poor ovarian responders? Ultrasound Obstet Gynecol 2018;52:530-4.

10. Liu C, Jiang $H$, Zhang W, Yin $H$. Double ovarian stimulation during the follicular and luteal phase in women $\geq 38$ years: a retrospective case-control study. Reprod Biomed Online 2017;35:678-84.

11. Cummins JM, Breen TM, Harrison KL, Shaw JM, Wilson LM, Hennessey JF. A formula for scoring human embryo growth rates in in vitro fertilization: its value in predicting pregnancy and in comparison with visual estimates of embryo quality. J In Vitro Fert Embryo Transf 1986;3:284-95.

12. Vladimirov IK, Tacheva D, Diez A. Theory about the embryo cryo-treatment. Reprod Med Biol 2017;16:118-25.

13. Shapiro BS, Daneshmand ST, Garner FC, Aguirre M, Hudson C. Clinical rationale for cryopreservation of entire embryo cohorts in lieu of fresh transfer. Fertil Steril 2014;102:3-9.

14. Horcajadas JA, Riesewijk A, Polman J, van Os R, Pellicer A, Mosselman $S$, et al. Effect of controlled ovarian hyperstimulation in IVF on endometrial gene expression profiles. Mol Hum Reprod 2005;11:195-205.
15. Ubaldi F, Bourgain C, Tournaye H, Smitz J, Van Steirteghem A, Devroey P. Endometrial evaluation by aspiration biopsy on the day of oocyte retrieval in the embryo transfer cycles in patients with serum progesterone rise during the follicular phase. Fertil Steril 1997;67:521-6.

16. Roque M, Lattes K, Serra S, Solà I, Geber S, Carreras R, et al. Fresh embryo transfer versus frozen embryo transfer in in vitro fertilization cycles: a systematic review and meta-analysis. Fertil Steril 2013;99:156-62.

17. Wei D, Liu JY, Sun Y, Shi Y, Zhang B, Liu JQ, et al. Frozen versus fresh single blastocyst transfer in ovulatory women: a multicentre, randomised controlled trial. Lancet 2019;393:1310-8.

18. Shapiro BS, Daneshmand ST, Garner FC, Aguirre M, Hudson C, Thomas S. Evidence of impaired endometrial receptivity after ovarian stimulation for in vitro fertilization: a prospective randomized trial comparing fresh and frozen-thawed embryo transfer in normal responders. Fertil Steril 2011;96:344-8.

19. Roque M, Valle M, Guimarães F, Sampaio M, Geber S. Freeze-all cycle for all normal responders? J Assist Reprod Genet 2017;34:179-85.

20. Dieamant FC, Petersen CG, Mauri AL, Comar V, Mattila $M$, Vagnini LD, et al. Fresh embryos versus freeze-all embryos - transfer strategies: nuances of a meta-analysis. JBRA Assist Reprod 2017;21:260-72.

21. Barnhart KT. Introduction: are we ready to eliminate the transfer of fresh embryos in in vitro fertilization? Fertil Steril 2014;102:1-2.

22. Garcia J, Jones GS, Acosta AA, Wright GL Jr. Corpus luteum function after follicle aspiration for oocyte retrieval. Fertil Steril 1981;36:565-72.

23. Kailasam C, Keay SD, Wilson P, Ford WC, Jenkins JM. Defining poor ovarian response during IVF cycles, in women aged $<40$ years, and its relationship with treatment outcome. Hum Reprod 2004;19:1544-7.

24. Hanoch J, Lavy Y, Holzer H, Hurwitz A, Simon A, Revel $A$, et al. Young low responders protected from untoward effects of reduced ovarian response. Fertil Steril 1998;69:1001-4.

25. Yin H, Jiang H, He R, Wang C, Zhu J, Cao Z. Cumulative live birth rate of advanced-age women more than 40 with or without poor ovarian response. Taiwan J Obstet Gynecol 2019;58:201-5.

26. Çelik S, Turgut NE, Yağmur E, Boynukalın K, Çelik DC, 


\section{Obstetrics \& Gynecology Science}

Vol. 64, No. 3, 2021

Fındıklı N, et al. The effects of fresh embryo transfers and elective frozen/thawed embryo transfers on pregancy outcomes in poor ovarian responders as defined by the Bologna criteria. Turk J Obstet Gynecol 2015; 12:132-8.

27. Spandorfer SD, Bendikson K, Dragisic K, Schattman G, Davis OK, Rosenwaks Z. Outcome of in vitro fertilization in women 45 years and older who use autologous oocytes. Fertil Steril 2007;87:74-6.

28. Tatone C, Amicarelli F, Carbone MC, Monteleone P, Caserta D, Marci R, et al. Cellular and molecular aspects of ovarian follicle ageing. Hum Reprod Update
2008; 14:131-42.

29. Erdem M, Erdem A, Biberoglu K, Arslan M. Age-related changes in ovarian volume, antral follicle counts and basal follicle stimulating hormone levels: comparison between fertile and infertile women. Gynecol Endocrinol 2003;17:199-205.

30. Vuong LN, Dang VQ, Ho TM, Huynh BG, Ha DT, Pham $T D$, et al. IVF transfer of fresh or frozen embryos in women without polycystic ovaries. N Engl J Med 2018;378:137-47.

31. Coutifaris C. "Freeze only"--An evolving standard in clinical in vitro fertilization. N Engl J Med 2016;375:577-9. 